\title{
A Tunable CMOS Resistor with Wide Tuning Range for Low Pass Filter Application
}

\author{
Desheng Ma, Bogdan M. Wilamowski, and Foster Fa Dai \\ Auburn University
}

\begin{abstract}
In this paper, a new tunable CMOS resistor is proposed. The resistance is inversely proportional to bias current, to provide the resistor with a wider tuning range. And transistors, composing the active resistor, work at saturation region to achieve very large resistance within a small area. As an example, a low pass RC filter using tunable CMOS resistor realized in $0.5-\mu \mathrm{m}$ CMOS technology is reported. The measured result shows that the cutoff frequency of low pass filter can be widely tuned from $5 \mathrm{kHz}$ to $1.9 \mathrm{MHz}$. The total harmonic distortion (THD) stays lower than $-40 \mathrm{~dB}$ for an input signal up to $100 \mathrm{mV} \mathrm{Vp}$-p at $500-\mathrm{kHz}$ input frequency. The die area of the low-pass filter is $0.25 \times 0.13 \mathrm{~mm}^{2}$ and the maximum power consumption (at $\mathrm{fc}=1.9 \mathrm{MHZ}$ ) is $5.7 \mathrm{~mW}$ with single 3.3-V power supply.
\end{abstract}

\section{INTRODUCTION}

Despite the effort to build all circuits in digital, analog filters are still highly needed in many applications owing to its low power consumption and simplicity. Tunable filtering is one of the open problems where tunable components, just like tunable resistors and capacitors, are very hard to be integrated. Recently, tunable filter design with tunable active devices is attracted more and more attention due to its lower power cost, smaller size and needless to say, higher level of integration. [1][2]

Two widely used tuning technologies for analog filters with tunable active components are the MOSFET-C filters and the transconductance- $\mathrm{C}$ filters (Gm-C filters). The primary drawback in each is subtle: For MOSFET resistor, the transistor works at triode region, it is very hard to get large resistance. [3][4] For Gm-C filter, due to the reason that $\mathrm{Gm}$ is proportional to square root of bias current, it has a limited tuning range.[5] Typically, the tuning range is lower than tens times. This drawback makes it unsuitable for applications where wide tuning range is needed.

In this paper, we present an improved tunable CMOS resistor with large resistance. And also the resistance is inversely proportional to bias current instead of square root of the bias current. Thus, the tuning range of cutoff frequency is much wider than $\mathrm{Gm}$ cell in $\mathrm{Gm}-\mathrm{C}$ filter. Analog filters utilize the tunable resistor we presented can overcome drawbacks stated above simultaneously. As an example, a second-order low pass RC filter using tunable CMOS resistor is proposed. The cutoff frequency can be widely tuned in the range of $5 \mathrm{kHz}$ to $1.9 \mathrm{MHz}$. The THD for a $500-\mathrm{kHz}$ input signal is lower than $-40 \mathrm{~dB}$ up to $100 \mathrm{mVpp}$ input signal amplitude.

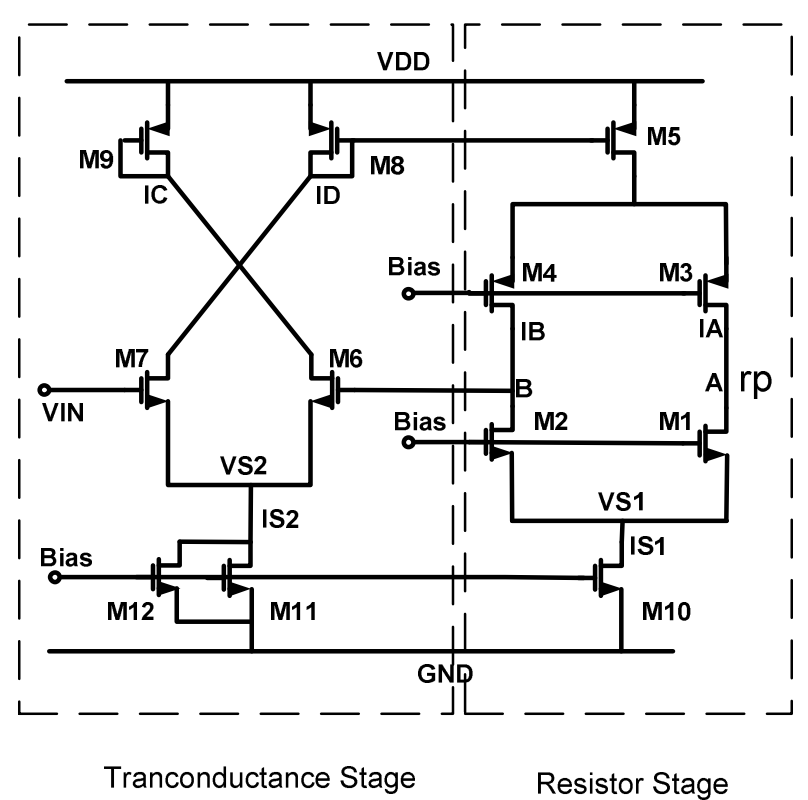

Fig. 1 Schematic of tunable resistor

\section{OPERATION THEORY OF TUNABLE FILTER}

\section{A. Tunable CMOS resistor}

Fig. 1 shows the conceptual schematic of tunable CMOS resistor. Tunable CMOS resistor can be seen as two parts: transconductance amplifier stage and resistor stage.

Differential pair M6 and M7 together with current mirror M9, M8, M5, and M10 12 constitute a transcondutance amplifier. This transconductance amplifier is loaded with internal cascade transistors. The gain can be described as follow:

$$
A_{V}=\frac{r_{o 4}\left(1+r_{o 5} \times g_{m 4}\right) \| r_{o 4}\left(1+r_{o 5} \times g_{m 4}\right)}{\frac{1}{g_{m 7}}+\frac{1}{g_{m 6}}}
$$

where $\mathrm{r}_{04,5}$ are the output resistances of transistors M4 and M5. $g_{\mathrm{m} 4,6,7}$ are the equivalent transconductances of transistor M4, M6 and M7. As shown, the voltage gain of this amplifier is very large. By connecting one input of the amplifier (gate of transistor M6) with its output (drain of transistor M4), it acts like a voltage follower. In this way, the voltage on $\mathrm{B}$ follows input voltage. Then the desired tunable resistance from VIN to output $\mathrm{A}$ is equal to resistance between point $\mathrm{A}$ and $\mathrm{B}$. The equivalent resistor model between point A and B is shown in Fig.2. 


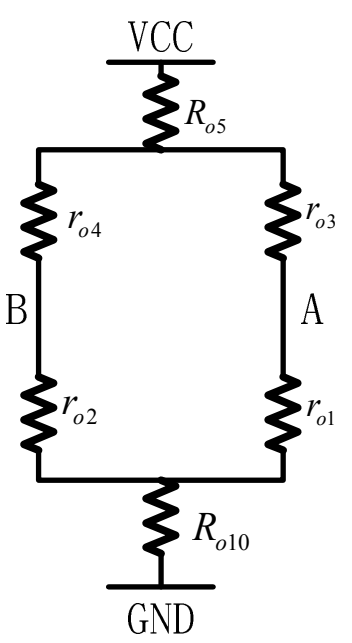

Fig. 2 Equivalent resistor model of resistor stage

To provide large resistance, transistor M1, M2, M3 and M4 work at saturation region in our design. As shown in Fig.2. Equivalent ground resistances $R_{o 10}$ and $R_{o 5}$ are very large compared to $\mathrm{r}_{01,2,3,4}$ and they can be neglected in the resistance calculation. Then the resistance between point $\mathrm{A}$ and $\mathrm{B}$ can be expressed as:

$$
\begin{aligned}
R_{A B} & =\left(r_{o 3}+r_{o 4}\right) \|\left(r_{o 1}+r_{o 2}\right) \\
& =\frac{1}{I_{\text {eff }}\left(\lambda_{n}+\lambda_{p}\right)}
\end{aligned}
$$

Where $r_{o 1,2,3,4}$ is the output resistance of transistors M1, M2, M3 and M4. $\lambda_{n}$ and $\lambda_{p}$ are channel length modulation parameters. And $\mathrm{I}_{\text {eff }}$ can be expressed as:

$$
I_{\text {eff }}=\frac{I_{A} \times I_{B}}{I_{A}+I_{B}}
$$

Due to symmetric design for the resistor stage, $I_{A}$ and $I_{B}$ are identical. Then the tunable CMOS resistor is achieved, the resistance equals to:

$$
R=\frac{1}{I_{S 1}\left(\frac{\lambda_{n}+\lambda_{p}}{2}\right)}
$$

As shown in equation (4), the resistance is inversely proportional to bias current $I_{S 1}$. It can be easily adjusted via changing transistor's bias current $\mathrm{I}_{\mathrm{S} 1}$ and $\mathrm{I}_{\mathrm{S} 2}$. Sweeps of tunable resistor are shown in Fig.3. In Fig.3, $\mathrm{V}_{\mathrm{T}}$ (x-axes) is the voltage adding on the tunable resistor and Iout (y-axis) is the current flow out from the tunable resistor. The bias current IS1 is shown on the top of Fig.3. The simulation result validates that the tunable resistance is inversely proportional to bias current. Fig 4 shows the resistance variation as bias current changing. Both resistance and bias current IS1 are in logarithm scale. As Fig.4 shown, the resistance can be tuned from $90 \mathrm{~K}$ ohm to $40 \mathrm{M}$ ohm by

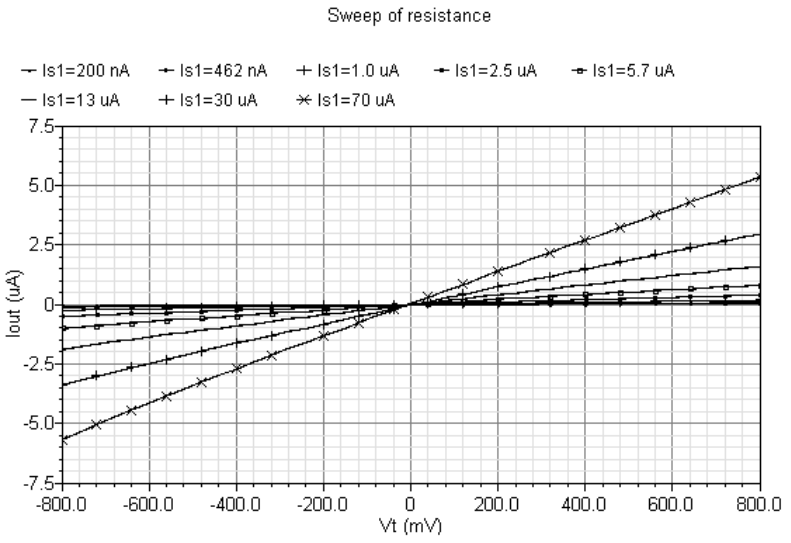

Fig. 3 Simulation result of sweeps of resistance Rus Is 1

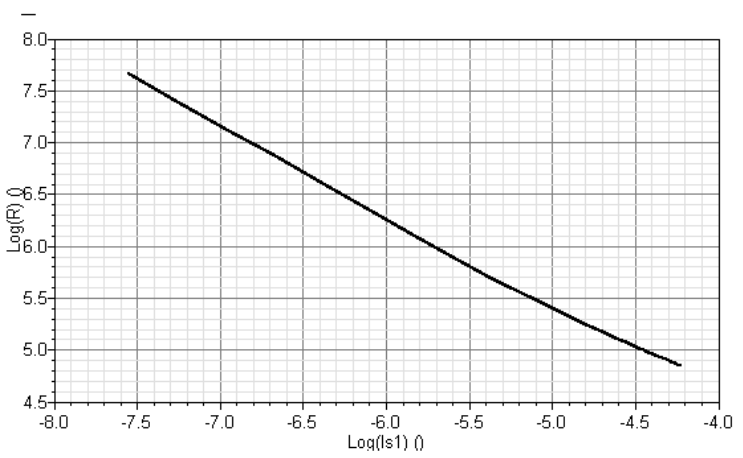

Fig. 4 Resistance variation as current changing (resistance and current are both in logarithm scale)

directly changing the bias current. Compared to traditional $\mathrm{Gm}$ cell, in which the tuning range is determined by square root of bias current, and MOSFET working in triode region to act as a resistor, the CMOS resistor in our design has much wider resistance tuning range and larger resistance.

Fig.5 shows the linearity performance of tunable resistor. Fig.5 (a) illustrates the harmonic distortion of tunable resistor under different frequency when output signal amplitude is held at $100 \mathrm{mV}$. As shown, the second harmonic distortion determines the THD, which is below $-48 \mathrm{~dB}$ when frequency is lower than $50 \mathrm{MHz}$. Fig.5 (b)

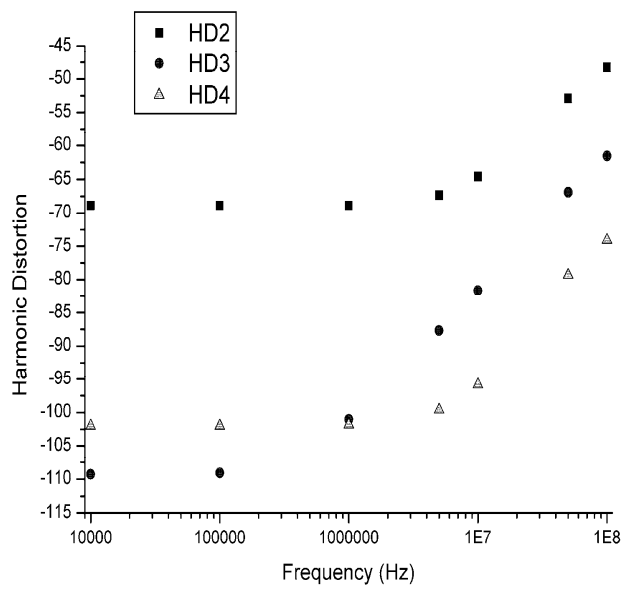

(a) 


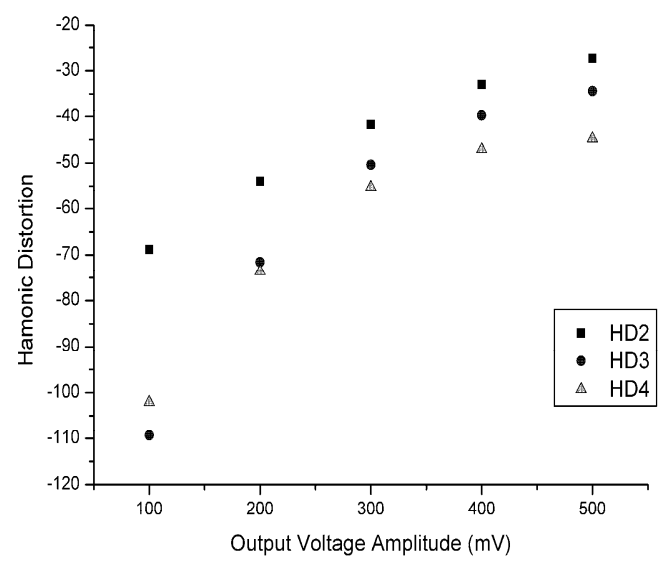

(b)

Fig.5. Experimental linearity results of tunable resistor for different frequency and different output power. (a) Harmonic distortion for different input signal frequency. (b) Harmonic distortion for different output signal amplitude

shows the harmonic distortion of tunable resistor as signal amplitude swept. The frequency of input signal is kept at $500 \mathrm{kHz}$. Both (a) and (b) show that second order harmonic distortion is the dominated nonlinearity factor. Thus, reduction of distortion can be obtained by using the tunable resistor in balanced structures.

\section{B. Low Pass Filter}

As mentioned above, the input impedance of the CMOS resistor is much larger than output impedance. Thus, they can be cascaded directly. A simple way to implement a second-order low-pass RC filter with proposed tunable resistor is shown in Fig.6. As shown, tunable resistors and capacitors are simply connected to compose a low-pass filter.

Since tunable resistor's pole frequency is far away from filter's pole frequency, its influence on low frequency pole can be neglected. Simple RC low-pass filter has a second order pole located at:

$$
p=-\omega_{c}=-\frac{1}{R C}=-\frac{I_{S 1}\left(\frac{\lambda_{n}+\lambda_{p}}{2}\right) I_{S 1}}{C}
$$

As shown above, the cut-off frequency can be directly tuning via changing bias current.

\section{IMPLEMENTATION OF TUNABLE FILTER IN VLSI TECHNOLOGY}

As an example, a second-order tunable low pass filter using the CMOS resistor as discussed above is implemented and measured. The circuit has been fabricated in $0.5-\mu \mathrm{m}$ CMOS technology. The conceptual schematic of low pass filter is shown in Fig.6. The total filter block occupies $0.25 \mathrm{~mm} \times 0.13 \mathrm{~mm}^{2}$ as shown in Fig.7. The tunable resistor block is as same as shown in Fig.1. Two tunable resistors in the filter are identical; differences will occur due to manufacturing tolerances. In case separate externally tuning is necessary.

To reduce influence of parasitic parameters and temperature, metal isolation metal (MIM) capacitor was chosen to implement capacitors $\mathrm{Ce} 1$ and $\mathrm{Ce} 2$.

\section{MEASURED RESULT}

The filter was packaged in $2 \mathrm{~mm} \times 2 \mathrm{~mm}$ QFN package. Fig. 8 shows the measured amplitude response of filter when input signal level is $300 \mathrm{mV} \mathrm{V} \mathrm{V}_{\mathrm{p}-\mathrm{p}}$. As shown, the cutoff frequency can be widely tuned from $5 \mathrm{kHz}$ to $1.9 \mathrm{MHz}$ as current control voltage changing from $0.75 \mathrm{~V}$ to $0.95 \mathrm{~V}$. The cutoff frequency is a little bit lower than simulation result due to the influence from parasitic capacitor of package and PCB board.

The linearity performance of the low pass filter is shown in Fig.9. The input signal frequency is set at $500 \mathrm{kHz}$ when cutoff frequency is at $1.5 \mathrm{MHz}$. The THD of the tunablefilter is $-40 \mathrm{~dB}$ when input amplitude is $100 \mathrm{mV} \mathrm{V}_{\mathrm{p}-\mathrm{p}}$ and $-34 \mathrm{~dB}$ when input amplitude is $200 \mathrm{mV} \mathrm{V} \mathrm{V}_{\mathrm{p}-\mathrm{p}}$.

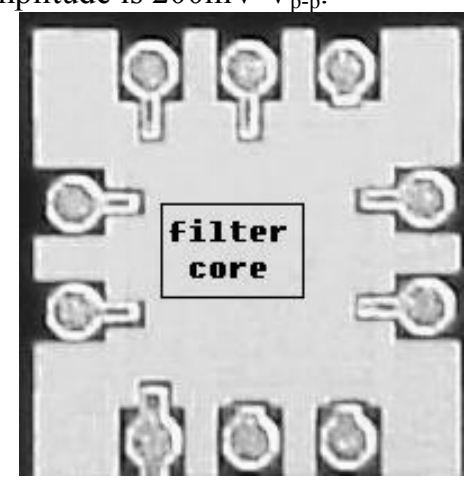

Fig. 7 Die photo of tunable low pass filter

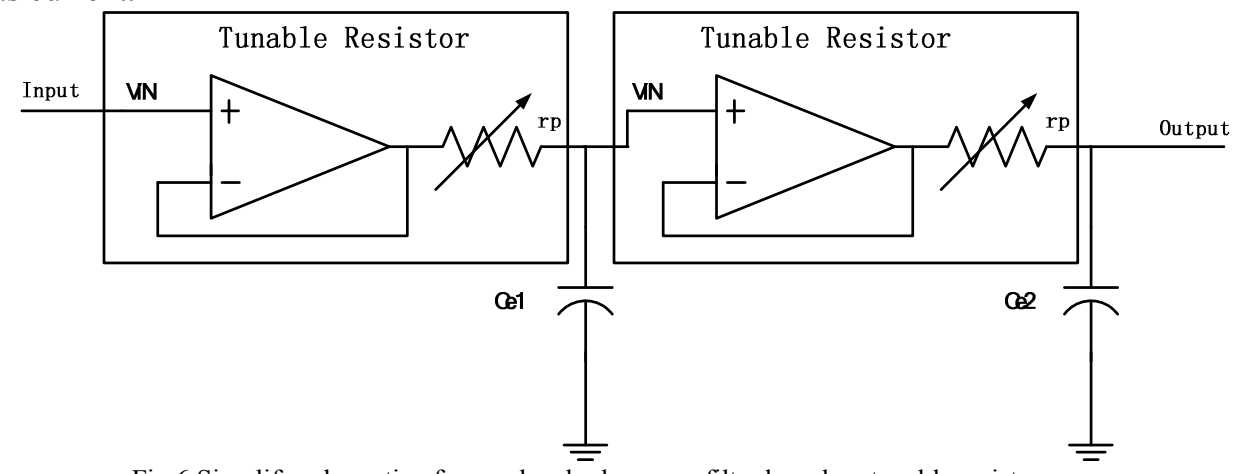

Fig.6 Simplify schematic of second-order low pass filter based on tunable resistor 


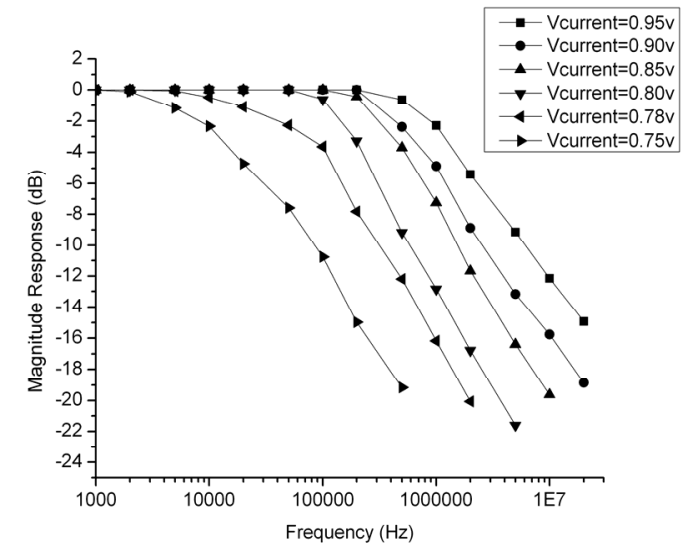

Fig.8. Measured amplitude response of tunable low pass

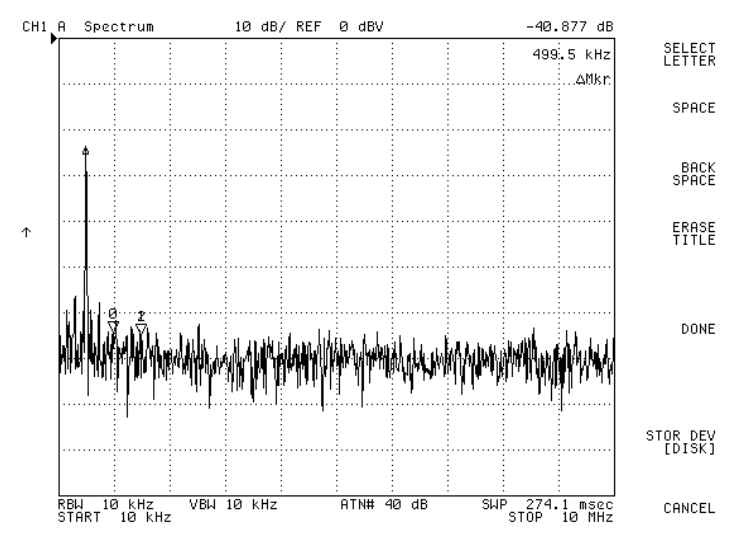

(a)

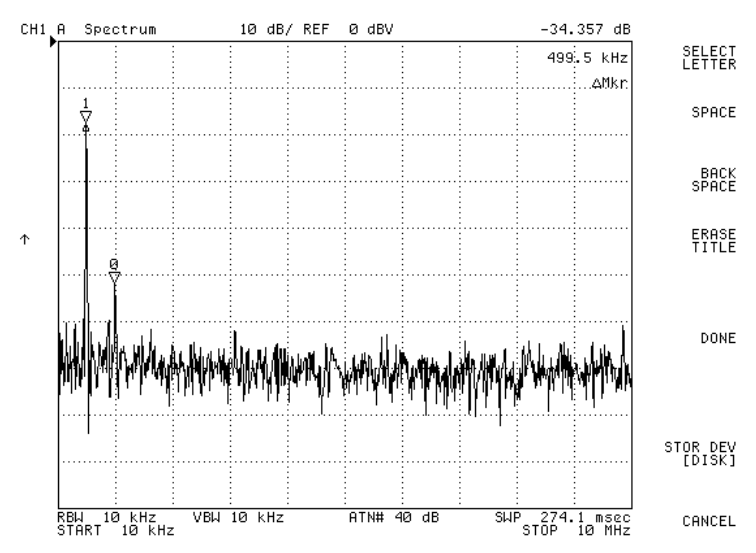

(b)

Fig.9. Measured output spectrum of the second-order low pass filter at: (a) input signal level of $100 \mathrm{mV} \mathrm{Vp-p} \mathrm{(b)} \mathrm{input} \mathrm{signal} \mathrm{level} \mathrm{of} 200 \mathrm{mV} \mathrm{Vp-p}$

A summary of the measured performance of the proposed filter is shown in Table I.
Table I. Summary of Measured Performance of the Proposed Filter

\begin{tabular}{|l|c|c|}
\hline & This work & Ref [2] \\
\hline Technology & $\begin{array}{c}0.5-\mu \mathrm{m} \\
\text { CMOS }\end{array}$ & $\begin{array}{c}0.18-\mu \mathrm{m} \\
\text { CMOS }\end{array}$ \\
\hline Power Supply & $3.3 \mathrm{~V}$ & $1.8 \mathrm{~V}$ \\
\hline Cutoff frequency range & $5 \mathrm{k}-1.9 \mathrm{M} \mathrm{Hz}$ & $0.5 \mathrm{M}-12 \mathrm{MHz}$ \\
\hline $\begin{array}{l}\text { Frequency tuning range } \\
\text { Freq_max/Freq_min }\end{array}$ & 380 & 24 \\
\hline $\begin{array}{l}\text { Power Consumption } \\
\text { (maximum cutoff frequency) }\end{array}$ & $5.7 \mathrm{~mW}$ & $4.7 \mathrm{~mW}$ \\
\hline $\begin{array}{l}\text { Power Consumption } \\
\text { (minimum cutoff frequency) }\end{array}$ & $2.8 \mathrm{~mW}$ & $1.1 \mathrm{~mW}$ \\
\hline $\begin{array}{l}\mathrm{f}-3 \mathrm{~dB} \text { Variation for Vcc 3.1V- } \\
3.8 \mathrm{~V}\end{array}$ & $\pm 3.4 \%$ & -- \\
\hline Core Size & $0.0325 \mathrm{~mm}^{2}$ & $0.125 \mathrm{~mm}^{2}$ \\
\hline
\end{tabular}

\section{CONCLUSION}

In this paper, a new CMOS tunable resistor has been designed and analyzed. Its main characteristics are: wide resistance tuning range, large resistance, low power consumption, and small die size. The resistance can be tuned by directly changing bias current. The performance of the proposed tunable resistor has been demonstrated by realizing a second-order low-pass RC filter in $0.5-\mu \mathrm{m}$ CMOS technology. The measured result shows that the cutoff frequency can be tuned from $5 \mathrm{kHz}$ to $1.9 \mathrm{MHz}$. The THD is $-40 \mathrm{~dB}$ at an input signal level of $100 \mathrm{mV} \mathrm{V}_{\mathrm{p}-\mathrm{p}}$. Although no automatically tuning was addressed in this paper, it is emphasized that automatic tuning schemes for the CMOS resistor we discussed are unavoidable.

\section{ACKNOWLEDGMENTS}

This work was supported under NASA Code ESR\&T program, contract number NNL05AA7C (ASTP-CCEI 2769). We would like to thank Guofu Niu, Wayne Johnson, Ben Blalock, Mohammad Mojarradi, Mike Palmer, and other team members for their contributions to this work.

\section{REFERENCES}

[1] Giacomino Bollati, Stefano Marchese, Marco Demicheli, and Rinaldo Castello, "An Eighth-Order CMOS Low-Pass Filter with $30-120 \mathrm{MHz}$ Tuning Range and Programmable Boost," IEEE J. Solid-State Circuit, vol. 36 July. 2001 pp. 1056-1066.

[2] Shinichi Hori, Tadashi Maeda, Noriaki Matsuno, and Hikaru Hida "Low-power widely tunable Gm-C filter with an adaptive DC-blocking, triode-biased MOSFET transconductor" Proc. Of the $30^{\text {th }}$ European Solid-State Circuits Conference, Leuven, Belgium, Sept. 2004

[3] E. Ozalevli and P. Hasler, "Design of a CMOS floating-gate resistor for highly linear amplifier and multiplier applications," Proc. Custom Integrated Circuits Conference, San Jose, California, Sept. 2005

[4] K. Vavelidis, Y. P. Tsividis, F. O. Eynde and Y. Papananos, "Sixterminal MOSFET's: modeling and applications in highly linear, electronically tunable resistors," IEEE J. Solid-State Circuit, vol. 32 Jan. 1997 pp. 4-12.

[5] Jun-Gi Jo; Changsik Yoo; Chunseok Jeong; Chan-Young Jeong; MiYoung Lee; Jong-Kee Kwon "A 1.2V, 10MHz, low-pass Gm-C filter with $\mathrm{Gm}$-cells based on triode-biased MOS and passive resistor in 0.13um CMOS technology" Proc. Custom Integrated Circuits Conference, San Jose, California, Sept. 2005 